\title{
Efecto del tratamiento térmico a baja temperatura para la obtención de películas delgadas de $\mathrm{SnO}$ \\ Effect of thermal annealing at low temperature for obtaining $\mathrm{SnO}$ thin films
}

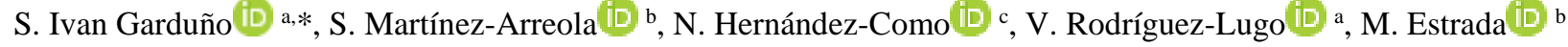 \\ a Área Académica de Ciencias de la Tierra y Materiales, Instituto de Ciencias Básicas e Ingeniería, UAEH, 42184, Mineral de la Reforma, Hidalgo, México. \\ ${ }^{\mathrm{b}}$ Sección de Electrónica del Estado Sólido, Departamento de Ingeniería Eléctrica, CINVESTAV-IPN, 07360, CDMX, México. \\ ${ }^{\mathrm{c}}$ Centro de Nanociencias y Micro y Nanotecnologías, Instituto Politécnico Nacional, 07360, CDMX, México.
}

\section{Resumen}

Por sus ventajas, el $\mathrm{SnO}$ es un óxido semiconductor (OMS) usado en transistores de película delgada (TFTs) de canal- $p$ y considerado para aplicaciones flexibles y transparentes. Aunque se deposita por varias técnicas, suele aplicarse un recocido $>200$ ${ }^{\circ} \mathrm{C}$ para obtenerlo, que es incompatible con la fabricación de TFTs con OMS tipo- $n$ y con sustratos flexibles y transparentes. Aquí mostramos el efecto de la presión parcial de oxígeno $\left(\mathrm{O}_{\mathrm{PP}}\right)$ y del recocido $\leq 20{ }^{\circ} \mathrm{C}$ en las propiedades estructurales, ópticas, composicionales y eléctricas de las capas depositadas por pulverización catódica. Una $\mathrm{O}_{\mathrm{PP}}$ al 9\% y un recocido entre 190 y 200 ${ }^{\circ} \mathrm{C}$ indujeron una mezcla de fases entre el estaño metálico y el $\mathrm{SnO}$ con orientación hacia diferentes planos, una transmitancia hasta de $36 \%$ en el espectro UV/Vis, un ancho de banda óptico de $\sim 2.83 \mathrm{eV}$ y una resistividad de $1 \mathrm{~m} \Omega \times \mathrm{cm}$. Esto coincide con los datos reportados del SnO tipo- $p$, lo que también posibilita su compatibilidad con la fabricación de TFTs con OMS tipo-n.

\section{Palabras Clave:}

Óxido de estaño, oxido semiconductor tipo- $p$, pulverización catódica reactiva, recocido a baja temperatura, electrónica flexible.

\begin{abstract}
Due to its advantages, the $\mathrm{SnO}$ is an oxide semiconductor (OMS) used in $p$-channel thin-film transistors (TFTs) and considered for flexible and transparent applications. Although it is deposited by several techniques, it is used to apply an annealing $>200{ }^{\circ} \mathrm{C}$ to obtain it, which is incompatible with the fabrication of TFTs with $n$-type OMS and with flexible and transparent substrates. Here we show the effect of the oxygen partial pressure $\left(\mathrm{O}_{\mathrm{PP}}\right)$ and the annealing $\leq 200{ }^{\circ} \mathrm{C}$ in the structural, optical, compositional, and electrical properties of the films deposited by sputtering. An $O_{\mathrm{PP}}$ at $9 \%$ and an annealing between 190 and $200^{\circ} \mathrm{C}$ induced a phases mixture of metallic tin and $\mathrm{SnO}$ with orientations to different planes, a transmittance up to $36 \%$ in the UV-Vis spectrum, an optical gap of $\sim 2.83 \mathrm{eV}$ and a resistivity of $1 \mathrm{~m} \Omega \times \mathrm{cm}$. This coincides with the reported data for the $p$-type $\mathrm{SnO}$, which also enables its compatibility with the fabrication of TFTs with $n$-type OMS.
\end{abstract}

Keywords:

Tin monoxide, $p$-type metal oxide semiconductor, reactive sputtering, low-temperature annealing, flexible electronics

\section{Introducción.}

Los óxidos metálicos semiconductores (OMS) han demostrado tener un gran potencial como capa activa para la fabricación de transistores de película delgada (TFTs) (Nomura et al., 2004). En gran medida, esto se debe a que es posible obtener películas delgadas de un OMS a baja temperatura $\left(<300^{\circ} \mathrm{C}\right)$ durante el proceso de fabricación del TFT, lo cual los hace compatibles con el desarrollo de aplicaciones de electrónica flexible y transparente dispuestas sobre áreas grandes; que pueden presentar una alta transmitancia óptica $(>80 \%)$, así como una alta movilidad de portadores de carga $(\geq 10$ $\mathrm{cm}^{2} \mathrm{~V}^{-1} \mathrm{~s}^{-1}$ ), comparada con la reportada para el silicio amorfo hidrogenado y los semiconductores orgánicos (Fortunato et al.,

\footnotetext{
*Autor para la correspondencia: salvador_garduno@uaeh.edu.mx

Correo electrónico: salvador_garduno@uaeh.edu.mx (Salvador Ivan Garduño), samuel_martinez@cinvestav.mx (Samuel Martínez-Arreola), nohernadezc@ipn.mx (Norberto Hernández-Como), venturar@uaeh.edu.mx (Ventura Rodríguez-Lugo), mestrada@yahoo.com (Magali Estrada)
} 
2012). Sin embargo, el desarrollo de aplicaciones de electrónica flexible y transparente se encuentra limitado por la obtención de tecnología complementaria; es decir, aquella que utiliza compuertas lógicas con TFTs de canal- $n$ y de canal- $p$. Esto se debe a que, a pesar de la realización de diferentes esfuerzos en la investigación sobre TFTs basados en OMS de conductividad tipo- $p$, éstos aún no cuentan con las características eléctricas que sean comparables con aquellas obtenidas en TFTs fabricados con OMS de conductividad tipo- $n$.

\subsection{Oxido de estaño tipo-p}

El óxido de estaño es un OMS policristalino que puede presentar conductividad tipo- $p$ o tipo- $n$ y un ancho de banda óptico desde $2.5 \mathrm{eV}$ hasta $3 \mathrm{eV}$, dependiendo de su estequiometria (De Barros, 2014). El tipo de conductividad de estas películas depende de las condiciones de depósito, por lo que existen diversas técnicas para el depósito de películas de óxido de estaño, entre las más comunes se encuentran: el depósito químico en fase de vapor, la pulverización catódica, el depósito por capas atómicas, el depósito por láser pulsado, entre otras. En general, el estaño metálico (Sn) puede oxidarse en dos diferente estados o fases como son: el dióxido de estaño $\left(\mathrm{SnO}_{2}\right)$ y el monóxido de estaño ( $\mathrm{SnO}$ ); lo cual depende estrechamente de las condiciones de depósito y del tratamiento térmico aplicado después de depositar la capa (Shan et al., 2016). El SnO presenta una mayor movilidad de huecos que otros OMS debido a la baja energía para la formación de vacancias de $\mathrm{Sn}$ (Nomura et al., 2011). Diferentes estudios sugieren que sólo la presencia de la fase del $\mathrm{SnO}$ tetragonal en el material depositado no necesariamente produce una mayor movilidad, pero una mezcla de ésta con la fase del Sn puede incrementar sustancialmente la movilidad de huecos (Caraveo et al., 2013). Dado que el $\mathrm{SnO}$ es una fase metaestable, la cual indica la probabilidad de que se reduzca a un estado de valencia menor, que resulta en el Sn, o que se oxide a un estado de valencia mayor, como es el $\mathrm{SnO}_{2}$, incluso en ausencia de un ambiente de oxígeno; de esta forma, el interés hacia el óxido de estaño ha incrementado debido a su potencial aplicación para el desarrollo de dispositivos electrónicos como uniones $p-n$ o transistores de película delgada, también su uso en electrodos de contacto transparentes, como sensores de gas y como catalizador de precursores para la obtención de ácidos (Batzill and Diebold, 2005), (Pan and Fu, 2001).

\subsection{Transistores de película delgada basados en SnO tipo-p}

Desde que se demostró en 2004 el uso de óxidos semiconductores metálicos en TFTs como capa activa (Nomura et al., 2004), ha incrementado el interés en desarrollar dispositivos basados en $\mathrm{SnO}$ con conductividad tipo- $p$ y su potencial implementación tanto en sustratos flexibles como en aplicaciones de electrónica transparente. Actualmente, los estudios sobre TFTs con OMS tipo- $p$ como capa activa han reportado habitualmente el uso del $\mathrm{SnO}$, utilizando distintas técnicas de depósito y la aplicación de un tratamiento térmico a una temperatura $>200{ }^{\circ} \mathrm{C}$, ya sea durante el proceso de fabricación del transistor o posterior a éste (Caraveo et al., 2013), (Fortunato et al., 2010), (Wu et al., 2019). Como se ha mencionado, el óxido de estaño es un material que presenta diferentes estados de oxidación, principalmente atribuido a la presión parcial de oxígeno empleada para su depósito y al tratamiento térmico posterior a éste, cuando se aplica alguna técnica de pulverización catódica. El control preciso de ambos factores resulta en una mezcla de fases de $\mathrm{Sn}$ y $\mathrm{SnO}$, lo que produce una conductividad tipo- $p$ y una alta movilidad de huecos (Caraveo et al., 2013). Adicionalmente, se ha reportado que la presencia y predominio de estos diferentes estados de oxidación del estaño pueden introducir modificaciones en las propiedades eléctricas, ópticas, morfológicas y estructurales de las películas delgadas depositadas por el método mencionado (Luo et al., 2012). Sin embargo, para el desarrollo de compuertas lógicas con tecnología complementaria basada en OMS, un proceso con una temperatura $>200{ }^{\circ} \mathrm{C}$ puede resultar incompatible con secuencias tecnológicas desarrolladas para la fabricación a baja temperatura de TFTs con OMS tipo- $n$ y, adicionalmente, con la mayoría de los sustratos utilizados en el desarrollo de la electrónica flexible y transparente (Park et al., 2019).

Hasta el momento, algunas limitantes adicionales reportadas en TFTs fabricados con SnO tipo- $p$ como capa activa, para su aplicación en tecnología complementaria, consisten en: una movilidad por efecto campo de huecos $\left(\mu_{F E}\right)$ con un valor máximo reportado de $6.4 \mathrm{~cm}^{2} / \mathrm{V} \cdot \mathrm{s}$, una relación entre las corrientes de encendido y apagado $\left(I_{O n} / I_{O f f}\right)<10^{5}$, una pendiente de subumbral (SS) $\sim 1 \mathrm{~V} / \mathrm{dec}$ y poco control sobre el voltaje de umbral, presentando tanto valores positivos como negativos (Caraveo et al., 2013), (Luo et al., 2012), (Wang et al., 2016), (Wu et al., 2019), (Zhong et al., 2016).

En este trabajo se utilizó la técnica de pulverización catódica reactiva por magnetrón de corriente directa (DC) para obtener películas delgadas a partir de un blanco de $\mathrm{Sn}$, las cuales se depositaron a temperatura ambiente y en una atmósfera de argón (Ar)/oxígeno $\left(\mathrm{O}_{2}\right)$ con presiones parciales de oxígeno al $7 \%, 9 \%$ y $10 \%$. Después, las capas fueron recocidas a una temperatura entre $160{ }^{\circ} \mathrm{C}$ y $200{ }^{\circ} \mathrm{C}$ en aire. Para analizar los cambios que inducen tanto la variación de la presión parcial de oxígeno $\left(\mathrm{O}_{\mathrm{PP}}\right)$ en el proceso de depósito, como el efecto del tratamiento térmico (TA) a baja temperatura $\left(\leq 200{ }^{\circ} \mathrm{C}\right)$, se realizó la caracterización estructural, óptica, composicional y eléctrica de las películas obtenidas. En primera instancia, este estudio evaluó la pertinencia de las condiciones de depósito y tratamiento térmico de las capas delgadas resultantes para su implementación en la obtención de estructuras metal-dieléctricosemiconductor de conductividad tipo- $p$, enfocando la compatibilidad del proceso con las secuencias tecnológicas desarrolladas para la fabricación de TFTs de canal- $n$. Por lo tanto, se aporta información relevante para la obtención de tecnología complementaria basada en OMS.

\section{Parte experimental.}

Se utilizaron sustratos de vidrio y silicio tipo- $p$ para el depósito de las películas delgadas y se limpiaron mediante un proceso similar al estándar RCA. El depósito a temperatura ambiente de las películas de $\mathrm{Sn}$ fue llevado a cabo mediante la técnica de pulverización catódica reactiva por magnetrón de DC. La distancia entre el objetivo y el sustrato se fijó a $16 \mathrm{~cm}$, mientras que el objetivo de Sn con una pureza de $99.999 \%$ tiene un tamaño de 3 pulgadas de diámetro. Antes del proceso de depósito, la cámara fue evacuada a una presión base de $10^{-6}$ Torr, la presión de depósito utilizada con un ambiente de argón/oxígeno $\left(\mathrm{P}_{\mathrm{Ar}}+\mathrm{P}_{\mathrm{O} 2}\right)$ y la potencia de DC aplicada fueron de 3 mTorr y $30 \mathrm{~W}$, respectivamente. Para evaluar los cambios en las propiedades estructurales, ópticas y eléctricas del proceso de depósito de las películas delgadas, se 
mantuvo un flujo de argón $\left(\mathrm{F}_{\mathrm{Ar}}\right)$ y un flujo de oxígeno $\left(\mathrm{F}_{\mathrm{O}}\right)$ que producen un cambio en la $\mathrm{O}_{\mathrm{PP}}$ del $7 \%, 9 \%$ y $10 \%$, donde $\mathrm{O}_{\mathrm{PP}}=\mathrm{F}_{\mathrm{O}} / \mathrm{F}_{\mathrm{Ar}}+\mathrm{F}_{\mathrm{O}}$. Justo después del proceso de depósito, las películas delgadas fueron sometidas a un TA a temperaturas entre 160 ${ }^{\circ} \mathrm{C}$ hasta $200{ }^{\circ} \mathrm{C}$ con incrementos de $10{ }^{\circ} \mathrm{C}$. Los TAs se realizaron en un ambiente de aire por una hora haciendo uso de una parrilla.

Las fases policristalinas de las películas depositadas y tratadas térmicamente fueron estudiadas por la técnica de difracción de rayos-x (XRD) con un equipo BRUKER D8 Advance usando un objetivo de cobre $(\mathrm{Cu}, 1.5416 \AA)$ con un voltaje de $40 \mathrm{kV}$ y una corriente de $30 \mathrm{~mA}$. La espectroscopia óptica fue usada para medir la transmitancia $(T)$ y ser analizadas a diferentes longitudes de onda desde el ultravioleta hasta aquella cercana al infrarrojo (desde $300 \mathrm{~nm}$ hasta $800 \mathrm{~nm}$ ). El espesor de las películas depositadas fue obtenido por medio de la técnica de reflectividad por rayos-x (XRR) y la técnica de elipsometría. Adicionalmente, la técnica de elipsometría fue usada para determinar el índice de refracción $(n)$ y el coeficiente de extinción (k) de las películas obtenidas. Un microscopio electrónico de barrido (SEM) con un detector para la espectroscopia por rayos-x de energía dispersiva (EDS) fue utilizado para realizar un análisis cuantitativo de los elementos que componen las películas analizadas. Finalmente, el método por cuatro puntas fue utilizado para medir la resistividad y obtener una tendencia sobre esta propiedad eléctrica por el efecto del recocido a diferentes temperaturas.

\section{Resultados y Discusión.}

\subsection{Caracterización estructural}

Los patrones de XRD de las películas tanto depositadas como tratadas térmicamente desde $160{ }^{\circ} \mathrm{C}$ hasta $200{ }^{\circ} \mathrm{C}$ para las diferentes $\mathrm{O}_{\mathrm{PP}}$ son presentadas en la Figura 1. Los patrones XRD indican que inicialmente existe un depósito de estaño policristalino, aunque con un proceso de oxidación incompleto (incorporación de oxígeno, parcialmente enlazado o no enlazado con el estaño) o con la presencia de $\mathrm{SnO}$ amorfo en las tres diferentes condiciones de $\mathrm{O}_{\mathrm{PP}}$ (Caraveo et al., 2013), (Fortunato et al., 2010). Además, los picos de difracción están relacionados con el Sn tetragonal (Carta ICDD No 00-0060395), donde aquellos de mayor intensidad muestran una orientación hacia los planos (200) y (101).

En las películas depositadas a un $7 \%$ de $\mathrm{O}_{\mathrm{PP}}$, tan pronto se aplica el TA, los patrones de XRD muestran también una estructura policristalina, pero cuyos picos de mayor intensidad no sólo coinciden con el Sn tetragonal, también corresponden a los planos (101) y (110) de la fase del SnO tetragonal (Carta ICDD No 00-006-0395). Adicionalmente, para las películas depositadas con $\mathrm{O}_{\mathrm{PP}}$ de $9 \%$ y $10 \%$, pero con TA, los picos de mayor intensidad también corresponden con el Sn y con el SnO tetragonales, orientados hacia los planos (200) y (101) del Sn, y los planos (101) y (110) del SnO. Este comportamiento es identificado en todos los casos en que las capas depositadas, bajo las condiciones previamente mencionadas, fueron recocidas, con excepción de la muestra recocida a $160^{\circ} \mathrm{C}$ y obtenida a $10 \%$ de $\mathrm{O}_{\mathrm{PP}}$, cuyo patrón se muestra en la Figura 1(III), la cual presenta picos de difracción relacionados al $\mathrm{SnO}$, pero de menor intensidad.

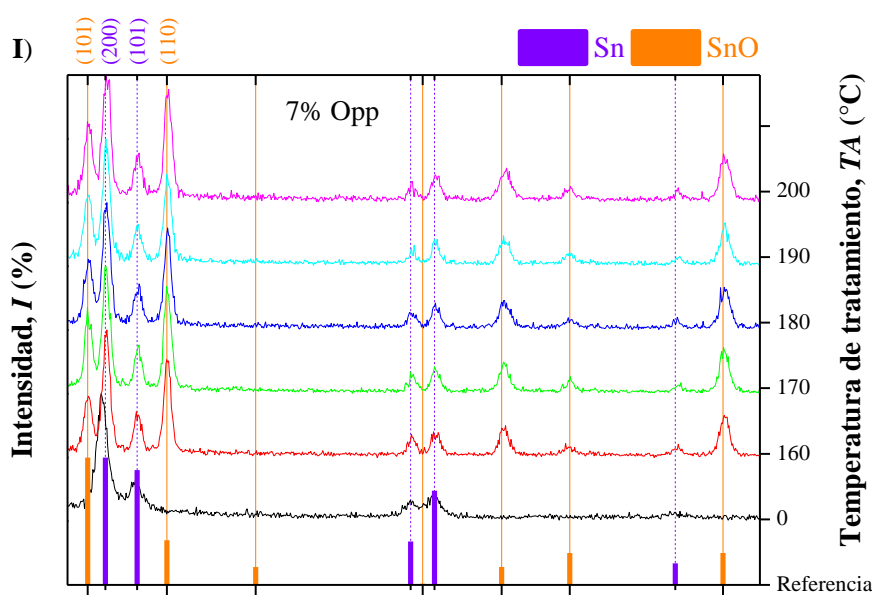

II)

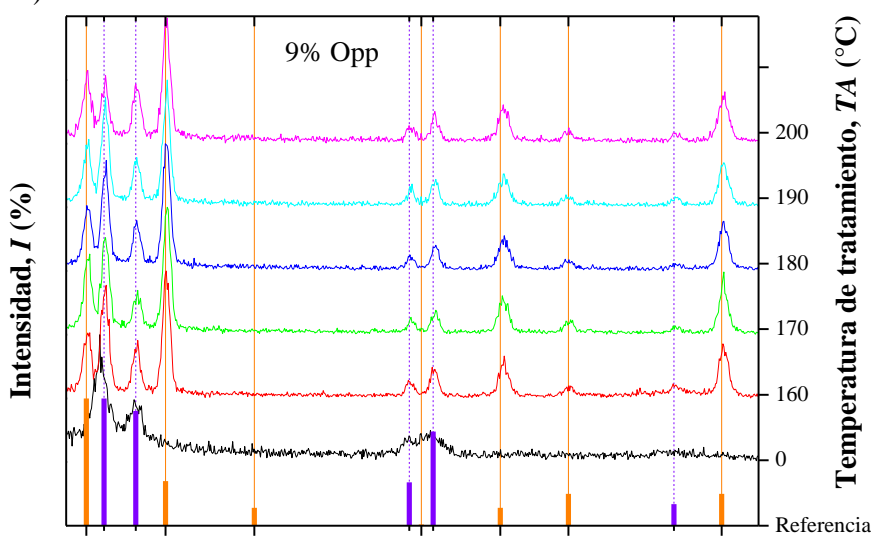

III)

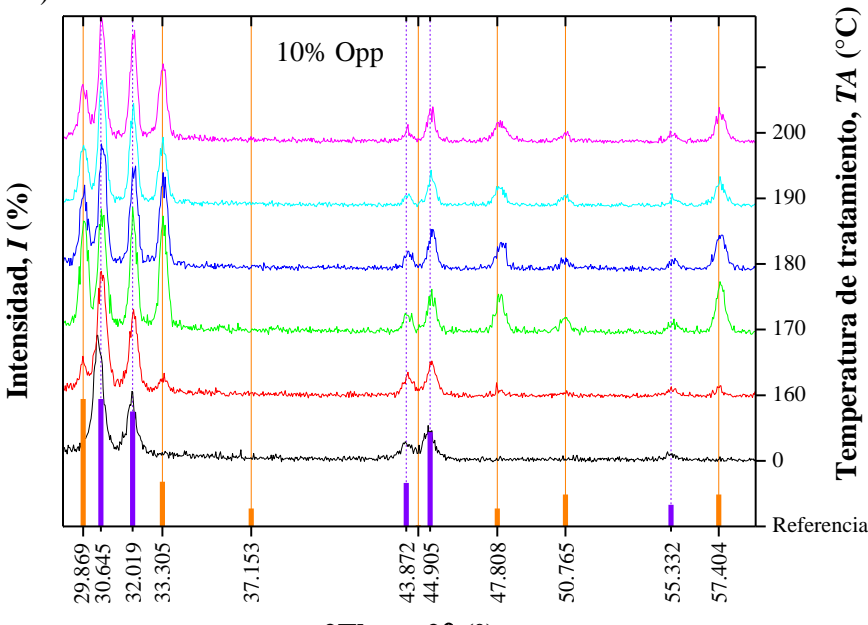

2Theta, $2 \theta\left(^{\circ}\right)$

Figura 1: Cartas cristalográficas de referencia para el Sn y el SnO tetragonales (barras). Patrones XRD de las películas de SnO depositadas con una OPp al 7\%, $9 \%$ y $10 \%$ (líneas continuas negras), $y$ aquellas con $\mathrm{TA}$ en el rango de temperatura de $160{ }^{\circ} \mathrm{C}$ a $200{ }^{\circ} \mathrm{C}$ (líneas continuas de color).

El resultado sobre esta mezcla de fases entre el $\mathrm{Sn}$ y el $\mathrm{SnO}$ coincide con diferentes estudios realizados, la cual ha sido atribuida a un proceso de oxidación incompleta y/o a la segregación del metal debido a una desproporción del oxígeno (Caraveo et al., 2013), (Fortunato et al., 2010), (Guzman et al., 2018), (Luo et al., 2012). Además, también ha sido demostrado que el aumento en la $\mathrm{O}_{\mathrm{PP}}$ puede generar una concentración de oxígeno suficiente para inducir la cristalización del Sn en una combinación de estados de oxidación; por ejemplo, incluyendo 
al tetraóxido de triestaño (Caraveo et al., 2013). Aunque en nuestro caso, la energía térmica aplicada es insuficiente para la

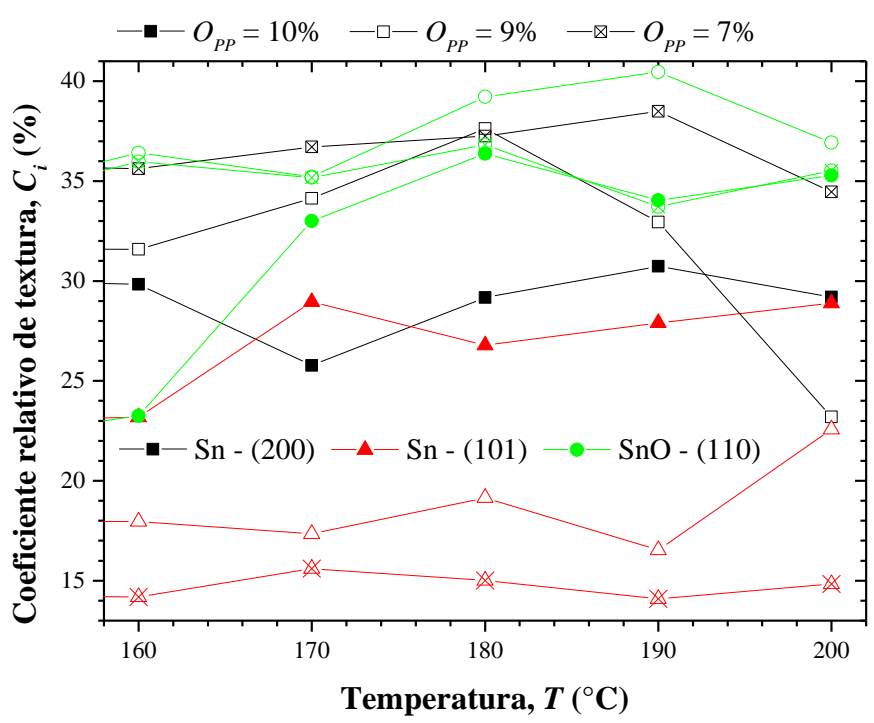

Figura 2: Variación del coeficiente de textura relativo en función de la OPP y del TA, sólo para los planos (200) y (101) del Sn, y el plano (110) del SnO. Los símbolos sólidos son para una OPP del 10\%, los vacíos para el $9 \%$ y aquellos con cruces para el $7 \%$.

formación de dicha composición, por lo cual, esto puede producir que los picos de difracción relacionados al $\mathrm{SnO}$ o a la combinación de otros óxidos sea de baja intensidad o indetectable por el equipo de medición (Caraveo et al., 2013).

En este sentido, en la Figura 1 también se observan diferencias en las intensidades de los picos de difracción vinculados al $\mathrm{Sn}$ y al SnO, considerando sólo los planos antes mencionados. Estas diferencias están relacionadas a distintas causas; por ejemplo, al tamaño y orientación del cristal, y a los defectos de la red atómica (Wang et al., 2016). Cabe mencionar que la única condición en que la fase del $\mathrm{SnO}$ es dominante corresponde a la capa depositada a $9 \%$ de $\mathrm{O}_{\mathrm{PP}}$ y $200^{\circ} \mathrm{C}$ de TA, la cual se observa en la Figura 1(II).

$\mathrm{Se}$ ha reportado que una fase de $\mathrm{SnO}$ puro exhibe una conductividad tipo- $p$; sin embargo, la presencia de esta mezcla de fases de $\mathrm{Sn}$ y $\mathrm{SnO}$ en la estructura de las películas delgadas, depositadas y recocidas bajo las condiciones antes mencionadas, no sólo permite obtener un material con características tipo- $p$, además contribuye al incremento de la movilidad de los huecos (Banerjee and Chattopadhyay, 2005), (Wang et al., 2016). De esta forma, tanto la formación de la fase del SnO, como la prevalencia de la fase del Sn en las capas obtenidas, dependen del control de la $\mathrm{O}_{\mathrm{PP}}$ y del TA en el rango entre 160 ${ }^{\circ} \mathrm{C}$ y $200{ }^{\circ} \mathrm{C}$.

Para cuantificar los planos de orientación de las películas obtenidas y para determinar las condiciones de procesamiento bajo las cuales alguna de las fases de $\mathrm{Sn}$ y $\mathrm{SnO}$ pueden ser predominante, se utilizó el coeficiente relativo de textura $\left(C_{i}\right)$, calculado a partir de la expresión (Guzman et al., 2018):

$$
C_{i}=100 \times \frac{I_{i} / I_{o i}}{\sum_{i=5}^{N}\left(I_{i} / I_{o i}\right)}
$$

donde $I_{i}$ e $I_{o i}$ se refieren a las intensidades máximas de los patrones XRD de las capas obtenidas y de intensidades respectivas al estándar de los materiales en las cartas cristalográficas de referencia, respectivamente. El término de la sumatoria resulta de los picos de difracción en los patrones XRD; donde $N$ es el número de picos observados dentro del rango de medición, el cual se igualó a 5 para todos los patrones correspondientes en la Figura 1.

En la Figura 2 se muestra la variación de $C_{i}$ con respecto al TA para las diferentes condiciones de $\mathrm{O}_{\mathrm{PP}}$, considerando sólo los planos (101) y (200) del Sn, y el plano (110) del SnO, debido a que los picos de difracción exhiben la mayor intensidad en dichos planos. Además, los picos de difracción que corresponden a los demás planos observados en la Figura 1 resultaron en valores de $C_{i}$ entre $25 \%$ y $10 \%$ para la fase del Sn, y entre $30 \%$ y $5 \%$ para la fase del $\mathrm{SnO}$.

Para el plano (200) del Sn, se observó un aumento de $C_{i}$ con la reducción de $\mathrm{O}_{\mathrm{PP}}$, dentro del rango de TA entre $160{ }^{\circ} \mathrm{C}$ y 190 ${ }^{\circ} \mathrm{C}$. Para el plano (101) del Sn, el $C_{i}$ se incrementa conforme aumenta la $\mathrm{O}_{\mathrm{PP}}$, lo cual se presentó para todas las temperaturas de recocido. Investigaciones previas han demostrado que al depositar películas delgadas de $\mathrm{SnO}$ por pulverización catódica reactiva, a mayor presión de depósito y mayor $\mathrm{O}_{\mathrm{PP}}$, se induce menor estrés en la red atómica, fomentando la cristalización orientada hacia el plano (101) del SnO (Caraveo et al., 2013), (Liu et al., 2015), (Wang et al., 2016); lo cual posiblemente también ocurre para la fase del Sn, pero hacia un determinado plano, como lo revela el comportamiento de $\mathrm{Ci}$ en la Figura 2.

Cuando se considera el plano (110) del $\mathrm{SnO}$, el $C_{i}$ se mantiene en un rango entre el $33 \%$ y el $41 \%$ para todas las condiciones de $\mathrm{O}_{\mathrm{PP}}$ y casi en todos los TA explorados. Un valor de $C_{i}$ del $23 \%$ fue obtenido sólo a $160{ }^{\circ} \mathrm{C}$ y $10 \%$ de OPP, lo cual coincide con las observaciones en los patrones XRD de la Figura 1 , donde una posible combinación de óxidos de estaño se ha formado, pero sin que ésta sea detectada por el equipo de medición. En el caso específico del $9 \%$ de $\mathrm{O}_{\mathrm{PP}}$, la aplicación de un TA a $200{ }^{\circ} \mathrm{C}$ produjo un $C_{i}$ de $\sim 23 \%$ para ambos planos del Sn considerados, pero para el plano (110) del SnO se obtuvo un $C_{i}$ del $37 \%$, indicando un predominio de la fase del $\mathrm{SnO}$ en la estructura de la capa obtenida.

\subsection{Caracterización óptica}

En la figura 3(I) se muestran los espectros de transmitancia óptica $(T r)$ en función de la longitud de onda $(\lambda)$, en un rango del espectro electromagnético correspondiente al ultravioleta/visible (UV/Vis), sólo para aquellas películas depositadas y tratadas térmicamente que presentaron la mayor transmitancia dentro del rango de medición. En la Figura 3 tampoco se consideraron los espectros de $\operatorname{Tr}$ de las películas que sólo fueron depositadas, debido a que éstas sólo presentaron la fase del Sn tetragonal, por lo que exhiben una alta reflectancia.

En el caso de las películas obtenidas al 7\% de OPP y con TA, el espectro de $\operatorname{Tr}$ muestra una variación desde el $17 \%$ hasta el $33 \%$, donde este máximo de $\operatorname{Tr}$ corresponde a un TA a $160{ }^{\circ} \mathrm{C}$. El comportamiento de la $T r$ está relacionado a los valores obtenidos de $C_{i}$ para los planos (200) del Sn y (110) del SnO, en la Figura 2. En ésta se observó que la orientación hacia el SnO es mayor que hacia el Sn sólo a $160^{\circ} \mathrm{C}$ y $200^{\circ} \mathrm{C}$. Además, 
conforme se incrementa el TA, el $C_{i}$ del plano (200) del Sn también aumenta, lo cual parece influir en una reducción de la $\operatorname{Tr}$ en la misma proporción, hasta que la temperatura del recocido es suficiente $\left(200^{\circ} \mathrm{C}\right)$ para que la estructura de la película sea orientada de nuevo hacia uno de los planos del $\mathrm{SnO}$ y la $\mathrm{Tr}$ aumenta otra vez, pero sin alcanzar el valor obtenido a $160^{\circ} \mathrm{C}$.

En las películas obtenidas al $9 \%$ de $\mathrm{O}_{\mathrm{PP}}$ y con TA, el espectro de $\operatorname{Tr}$ se mantiene hasta que se aplica un TA de $200{ }^{\circ} \mathrm{C}$ y se produce un incremento en $\operatorname{Tr}$ hasta alcanzar un máximo de $\sim 36 \%$ en la región del espectro UV/Vis medida. Este comportamiento también podría estar relacionado con la tendencia que presenta el $C_{i}$ correspondiente, donde los valores asociados al plano (110) del SnO son ligeramente mayores que para el plano (200) de la fase del Sn $\left(C_{i} \leq 37 \%\right)$ bajo las diferentes temperaturas de recocido aplicadas. Cuando se aplica un TA a $200{ }^{\circ} \mathrm{C}$, el $C_{i}$ para ambos planos considerados del $\mathrm{Sn}$ resulta en un valor del $23 \%$ y se observa un incremento en la $\operatorname{Tr}$ en el rango de $\lambda$ desde $350 \mathrm{~nm}$ hasta $800 \mathrm{~nm}$.

Para las películas obtenidas al $10 \%$ de OPP y con TA, el espectro de $\operatorname{Tr}$ muestra los valores mínimos para las tres diferentes condiciones de $\mathrm{O}_{\mathrm{PP}}$ utilizadas, con máximos que varían entre el $11 \%$ y el $17 \%$ para el rango de $\lambda$ de $550 \mathrm{~nm}$ a650 nm. Aunque la variación en la $\operatorname{Tr}$ muestra una cierta tendencia acorde al comportamiento del $C_{i}$ para el plano (110) del SnO en la Figura 2, debido al incremento en la orientación hacia el plano (101) del Sn $\left(C_{i}>26 \%\right)$, al parecer la $\operatorname{Tr}$ no alcanza a superar el $20 \%$ en las películas con TA entre $170{ }^{\circ} \mathrm{C}$ y $200{ }^{\circ} \mathrm{C}$. Además, se observa que la diferencia entre los valores del $C_{i}$ para los planos considerados del Sn y del SnO podría influir en el espectro de la $\operatorname{Tr}$ obtenido; es decir:

- Cuando $\left[C_{i(\mathrm{SnO})}-C_{i(\mathrm{Sn})}\right]<5 \%$, la $\operatorname{Tr}$ no supera el $12 \%$;

- Cuando $\left[C_{i(\mathrm{SnO})}-C_{i(\mathrm{Sn})}\right]>5 \%$, la $\operatorname{Tr}$ alcanza valores por encima del $12 \%$.

De esta forma, esto también podría explicar que la $\operatorname{Tr}$ medida en la película tratada a $180{ }^{\circ} \mathrm{C}$ exhibe valores mayores que aquella con un TA a $200{ }^{\circ} \mathrm{C}$, dentro de la región del espectro UV/Vis analizada. Esto debido a que la diferencia mencionada es de $\sim 8 \%$, para el caso del TA a $180{ }^{\circ} \mathrm{C}$, mientras que una diferencia de $\sim 6 \%$ corresponde al caso de TA a $200{ }^{\circ} \mathrm{C}$. El ancho óptico de la banda prohibida $\left(E_{g}\right)$ fue determinado en la región de mayor absorción, extrapolando la región lineal del gráfico de $(\alpha \times h v)^{2}$ con respecto a la energía del fotón $(h v)$ (Tauc and Menth, 1972), a partir de los espectros de $\operatorname{Tr}$ para todas las películas con TA. Sin embargo, en la Figura 3 (II) sólo se muestran los gráficos de Tauc para las capas que exhibieron una mayor $\operatorname{Tr}$ dentro de la región del espectro UV/Vis medida, bajo las diferentes condiciones de $\mathrm{O}_{\mathrm{PP}} \mathrm{y}$ de TA.

En general, el $E_{g}$ para las películas depositadas es menor en comparación con las películas recocidas, ya que se encuentra presente la fase del Sn como dominante, actuando como películas metálicas altamente reflectivas, por lo que el ancho de banda se encuentra en valores cercanos a $\sim 0.7 \mathrm{eV}$ como los ya reportados para esta condición (Wang et al., 2016). El $E_{g}$ obtenido para las películas que presentan la fase de $\mathrm{SnO}$ varía entre un valor de $2.69 \mathrm{eV}$ a $2.83 \mathrm{eV}$ a razón del aumento de la OPP. Donde el valor mínimo corresponde al proceso de depósito con una $\mathrm{O}_{\mathrm{PP}}$ del $10 \%$, mientras que el valor máximo fue extraído para una condición de depósito del 9\%. Los valores obtenidos de $E_{g}$ concuerdan con aquellos reportados para el
SnO tetragonal, de $2.5 \mathrm{eV}$ a $3.0 \mathrm{eV}$ (Guzman et al., 2018), (Yang et al., 2015).
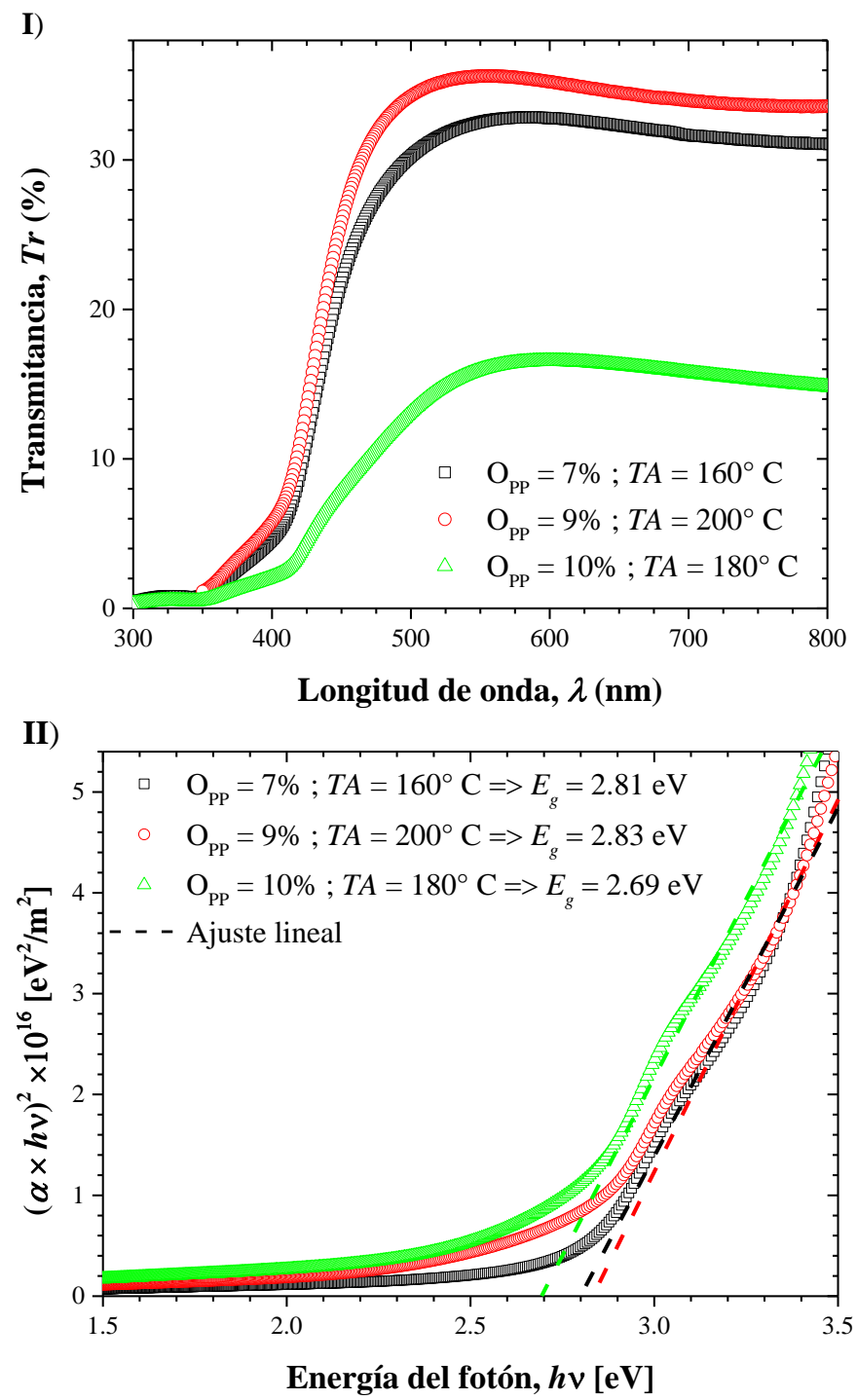

Figura 3: I) Espectro de transmitancia óptica y II) Gráfico de $(\alpha \times h v)^{2}$ con respecto a la energía del fotón $(h v)$ de las películas que presentan un máximo para cada $O_{P P}$.

La variación del $E_{g}$ en las películas depositadas y recocidas también podría estar relacionada con los resultados de los patrones XRD y el comportamiento del $C_{i}$, donde la presencia de la fase cristalina del $\mathrm{SnO}$ en la estructura del material induce cambios notables en sus propiedades ópticas, incluso en el rango visible del espectro electromagnético. Esto es, una mayor contribución en la orientación hacia los planos del Sn produce una reducción en la $\operatorname{Tr}$ y en el $E_{g}$, lo cual puede deberse a que la fase cristalina del Sn se comporta como partículas metálicas que tienden a incrementar la reflectividad de la superficie de las películas obtenidas (Wang et al., 2016). Sin embargo, se requiere realizar un mayor número de muestras y las mediciones correspondientes, un análisis más detallado para justificar las tendencias que fueron observadas y encontrar la relación entre los resultados obtenidos mediante la caracterización por XRD y aquella realizada por espectrofotometría, e incluso con el espesor de la capa. 
Tabla 1: Variación del índice de refracción $(n)$ y el coeficiente de extinción $(k)$ en función de O rente $\lambda$.

\begin{tabular}{ccccccccccccc}
\hline OpP & \multicolumn{4}{c}{$\mathbf{7 \%}$} & \multicolumn{4}{c}{$\mathbf{9 \%}$} & \multicolumn{3}{c}{$\mathbf{1 0 \%}$} \\
\hline Longitud de Onda & $632.8 \mathrm{~nm}$ & $824.3 \mathrm{~nm}$ & $632.8 \mathrm{~nm}$ & $824.3 \mathrm{~nm}$ & $632.8 \mathrm{~nm}$ & $824.3 \mathrm{~nm}$ \\
\hline Parámetro óptico & $n$ & $-k$ & $n$ & $-k$ & $n$ & $-k$ & $n$ & $-k$ & $n$ & $-k$ & $n$ & $-k$ \\
\hline Depositada & 3.29 & 2.42 & 3.83 & 1.55 & 3.56 & 2.43 & 4.09 & 1.55 & 3.25 & 2.9 & 4.3 & 2.3 \\
\hline & 2.02 & 0.003 & 1.73 & & 1.95 & 0.04 & 1.73 & & 2.02 & 0.002 & 1.80 \\
Recocida desde $160^{\circ} \mathrm{C}$ hasta & $\mathbf{\pm}$ & \pm & \pm & 0 & \pm & \pm & \pm & 0 & \pm & \pm & \pm & 0 \\
$200{ }^{\circ} \mathrm{C}$ & 0.02 & 0.002 & 0.02 & & 0.02 & 0.02 & 0.02 & & 0.03 & 0.001 & 0.02 & \\
\hline
\end{tabular}

Mediante la técnica de elipsometría se determinó el espesor de las películas, obteniéndose un valor de $66 \pm 4 \mathrm{~nm}$ para las capas depositadas con un $7 \%$ de $\mathrm{O}_{\mathrm{PP}}$, un promedio de $75 \pm 5 \mathrm{~nm}$ con un $9 \%$ y un espesor de $70 \pm 5 \mathrm{~nm}$ bajo una condición del $10 \%$. Considerando esta técnica, también fueron estimados los parámetros del índice de refracción ( $n$ ) y el coeficiente de extinción $(k)$ de dichas películas delgadas, los cuales han sido enlistados en la Tabla 1.

Para aquellas películas que sólo han sido depositadas, cabe destacar que los valores determinados de $n$ y $k$ son diferentes a los valores reportados para el $\mathrm{Sn}, n \sim 2.38$ y $k \sim 4.73$ a una $\lambda=632.8 \mathrm{~nm}$ (Golovashkin and Motulevich, 1965). Esto puede deberse al contenido de oxígeno que exhiben las películas desde su depósito, acorde a los resultados exhibidos por las mediciones de EDS. Específicamente para la capa depositada a $10 \%$ de $\mathrm{O}_{\text {PP }}$ y recocida a $160{ }^{\circ} \mathrm{C}$, también se obtuvieron los parámetros ópticos correspondientes a las capas sin TA, lo cual se debe al predominio de la fase del Sn en la estructura de la capa, como lo han demostrado los resultados de XRD. Cuando se realiza el TA, independientemente de la OPP y de la temperatura aplicadas, los parámetros $n$ y $k$ disminuyen hasta alcanzar valores cercanos a los reportados previamente para el $\mathrm{SnO}$ policristalino (Baco et al., 2012), (Liang et al., 2010), (Luo et $a l ., 2012)$. A pesar de que las películas delgadas obtenidas presentan la fase del $\mathrm{Sn}$, los resultados sugieren que el TA posterior al depósito logra inducir un cambio en las capas hasta que adquieren las propiedades ópticas del $\mathrm{SnO}$, aunque el recocido haya sido desarrollado en un rango de baja temperatura.

Mediante la técnica de XRR se confirmó el espesor de las películas obtenidas, determinado previamente por medio de elipsometría. Una vez realizadas las mediciones de XRR, se simuló el comportamiento de los datos experimentales para extraer la información pertinente. Los valores del espesor obtenidos resultaron muy cercanos a aquellos determinados por la técnica de elipsometría; es decir, para un 7\% de $\mathrm{O}_{\mathrm{PP}}$ el espesor resultó de $70.3 \mathrm{~nm}$ en promedio, para el $9 \%$ se obtuvo un promedio de $76.5 \mathrm{~nm}$ y, finalmente, un valor promedio de $74.7 \mathrm{~nm}$ correspondió con el $10 \%$ de $\mathrm{O}_{\mathrm{PP}}$, lo cual permite determinar con mayor precisión la razón de depósito de las películas delgadas bajo las diferentes condiciones consideradas. Tanto las capas depositadas como aquellas con TA mostraron una densidad $(\rho)$ que varía desde $6.1 \mathrm{gr} / \mathrm{cm}^{3}$ hasta $6.35 \mathrm{gr} / \mathrm{cm}^{3}$, estos valores son muy cercanos a la $\rho$ de $6.45 \mathrm{gr} / \mathrm{cm}^{3}$ reportada para el $\mathrm{SnO}$, mientras que para el dióxido de estaño $\left(\mathrm{SnO}_{2}\right)$ se ha establecido un valor de $6.95 \mathrm{gr} / \mathrm{cm}^{3}$ y el $\mathrm{Sn}$ tiene una $\rho$ de 7.2 $\mathrm{gr} / \mathrm{cm}^{3}$ (Allen et al., 2013), (Meyer et al., 2001). Aquellas películas sin TA también exhibieron un valor de $\rho$ cercano al reportado para el SnO. Este resultado también sugiere la oxidación incompleta del $\mathrm{Sn}$ o la presencia de $\mathrm{SnO}$ amorfo posterior al proceso de depósito, que no depende de la $\mathrm{O}_{\mathrm{PP}}$ en el rango considerado.

\subsection{Caracterización composicional}

Utilizando la técnica de EDS, se estudió la composición únicamente de las películas que fueron depositadas a diferente condición de $\mathrm{O}_{\mathrm{PP}}$ y las capas con TA a $200{ }^{\circ} \mathrm{C}$.

Las imágenes del SEM (Figura A del Apéndice A) mostraron gránulos (regiones claras) y surcos diminutos (regiones oscuras) que se hacen más evidentes e incrementan su tamaño conforme aumenta el porcentaje de $\mathrm{O}_{\mathrm{PP}}$ cuando se aplica el TA, además, la rugosidad presenta un incremento acorde a esta misma variación de parámetros. Esto coincide con los resultados de la rugosidad, determinados a través de la simulación de las mediciones de XRR, cuyos valores promedio fueron de $\sim 0.69 \mathrm{~nm}, \sim 0.75 \mathrm{~nm}$ y $\sim 0.82 \mathrm{~nm}$, producidos por el TA a las diferentes temperaturas y condiciones de $\mathrm{O}_{\mathrm{PP}}$ al 7\%, $9 \%$ y $10 \%$, respectivamente. Se ha demostrado que en las películas delgadas de $\mathrm{SnO}$, depositadas por pulverización catódica reactiva a una presión de depósito >2 mTorr, la rugosidad puede incrementar rápidamente desde un valor cuadrático medio (RMS) igual a $\sim 0.5 \mathrm{~nm}$, para un recocido a $150{ }^{\circ} \mathrm{C}$, hasta $\sim 3.5 \mathrm{~nm}$ al formarse la fase del $\mathrm{SnO}$ policristalino con un proceso de TA a $175^{\circ} \mathrm{C}$ (Li et al., 2018). Además, en dicho trabajo las imágenes del SEM de las capas de $\mathrm{SnO}$ también muestran la presencia de regiones claras y oscuras, las cuales fueron atribuidas a la formación del $\mathrm{SnO}$ policristalino, y sus fronteras de grano que, a su vez, habilitan el crecimiento de cristales de Sn a través de estas fronteras al incrementar la temperatura del recocido (Li et al., 2018).

La Figura 4 muestra los porcentajes atómicos del Sn y del oxígeno $(\mathrm{O})$ contenidos en las películas que sólo fueron depositadas y aquellas que fueron recocidas a $200^{\circ} \mathrm{C}$, con respecto a la variación de la $\mathrm{O}_{\mathrm{PP}}$. Es notable que el contenido de $\mathrm{Sn}$ incrementa acorde al aumento en el porcentaje de la $\mathrm{O}_{\mathrm{PP}}$ utilizada, variando desde $\sim 6$ at $\%$ a $\sim 9$ at $\%$ para ambas condiciones de procesamiento. Además, el contenido de O presenta un incremento entre el $7 \%$ y el $9 \%$ de $\mathrm{O}_{\mathrm{PP}}$, pero se observa una reducción importante en el contenido de $\mathrm{O}$ cuando la $\mathrm{O}_{\mathrm{PP}}$ aumenta al $10 \%$, alcanzando $\sim 8$ at $\%$ en la película que no ha sido tratada térmicamente. Esto exhibe un contenido de $\mathrm{O}$ que supera al de Sn lo suficiente para considerar que cada átomo de $\mathrm{Sn}$ tiene la posibilidad de enlazarse con un átomo de $\mathrm{O}$ y alcanzar la estequiometría del $\mathrm{SnO}$. Con excepción de la capa que fue obtenida al $10 \%$ de $\mathrm{O}_{\mathrm{PP}}$ y sin aplicarle un TA, donde el porcentaje atómico de $\mathrm{O}$ es menor que aquel del $\mathrm{Sn}$ (proceso de oxidación incompleta del $\mathrm{Sn}$ ). Estos resultados explican las 
características presentadas de las capas que no fueron tratadas térmicamente; es decir, bajo las condiciones del proceso de depósito es posible obtener películas delgadas, ya sea con una mezcla entre la fase del Sn y la fase de $\mathrm{SnO}$ amorfo, o de $\mathrm{Sn}$ oxidado parcialmente.

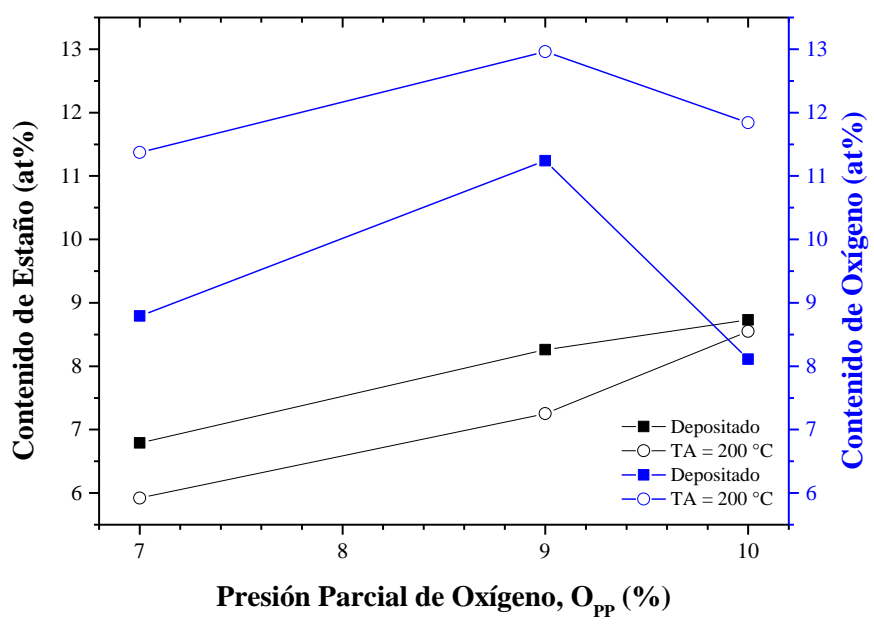

Figura 4: Gráfico del contenido de $S n$ y $O$ con respecto a la OpP en las películas sin TA y aquellas recocidas a $200{ }^{\circ} \mathrm{C}$.

En general, un proceso de recocido a baja temperatura $\left(\leq 200{ }^{\circ} \mathrm{C}\right)$ aplicado a las capas depositadas puede proveer la energía suficiente a su estructura para la cristalización del SnO. Además, este tipo de TA hasta una temperatura de $200{ }^{\circ} \mathrm{C}$ puede producir un incremento en el contenido de $\mathrm{O}$ en las películas depositadas; es decir, se realiza un proceso de difusión del oxígeno para la formación de una estructura policristalina con una mezcla de fases entre el Sn y el SnO, como lo exhiben el gráfico de la Figura 4 y los patrones XRD en la Figura 1.

\subsection{Caracterización eléctrica}

Finalmente, se realizó la medición de la resistividad eléctrica $\left(\rho_{e}\right)$ por la técnica de cuatro puntas. El proceso de medición consistió en la aplicación de una corriente $(I)$ con polarización positiva y la medición del voltaje $(V)$ generado; después, se aplicó una $I$ con la misma magnitud, pero de polarización negativa, y la medición correspondiente de la caída de potencial producida, repitiendo cada etapa de medición cinco veces más y con dos diferentes magnitudes de la $I$, de $10 \mu \mathrm{A}$ y 50 $\mu \mathrm{A}$ para las capas que sólo fueron depositadas, y de $1 \mathrm{~mA}$ y 10 $\mathrm{mA}$ para las capas que fueron recocidas. El valor de la $\rho_{e}$ se determina mediante (2) y se obtiene un valor promedio para cada $I$, utilizando el valor absoluto de la $I$ y $V$ :

$$
\rho_{e}=\frac{V}{I} \times \frac{t \times \pi}{\ln (2)}
$$

donde $t$ es el espesor de la capa obtenida.

Siguiendo el procedimiento descrito, en la Figura 5 se observa el comportamiento de $\rho_{e}$ con respecto a la temperatura del TA para cada una de las condiciones de OPP utilizadas durante el depósito de las capas.

En las películas sin TA se ha observado que la $\rho_{e}$ presenta una reducción conforme se incrementa la $\mathrm{O}_{\mathrm{PP}}$. Para las condiciones de $7 \%$ y $9 \%$ de $O_{\mathrm{PP}}$, el valor de la $\rho_{e}$ es de $\sim 1 \Omega \times \mathrm{cm}$, mientras que al incrementar dicha presión al $10 \%$, la $\rho_{e}$ se reduce casi en dos órdenes de magnitud hasta un valor de $20 \mathrm{~m} \Omega \times \mathrm{cm}$. Los valores resultantes de $\rho_{e}$ son bastante cercanos a los reportados en las películas de $\mathrm{SnO}$ obtenidas bajo una condición de baja presión de depósito ( $\leq 2 \mathrm{mT}$ Torr) y una $\mathrm{O}_{\mathrm{PP}}$ en el rango entre $7 \%$ y $11 \%$ (Fortunato et al., 2010). Esta reducción de la $\rho_{e}$, con el incremento de la $\mathrm{O}_{\mathrm{PP}}$, es una consecuencia de la presencia de un mayor contenido de $\mathrm{Sn}$ que de O, como se observa en la Figura 4, donde el Sn provee a la capa una propiedad más conductiva. Este comportamiento de la $\rho_{e}$ con respecto al incremento de la $\mathrm{O}_{\mathrm{PP}}$ parece seguir la misma tendencia después del TA aplicado a una temperatura entre 160 y $180{ }^{\circ} \mathrm{C}$.

Una $\rho_{e}$ típica para el $\mathrm{Sn}$ es de $12 \mu \Omega \times \mathrm{cm}$ (Lorenz et al., 2016), por lo que una resistividad entre $20 \mathrm{~m} \Omega \times \mathrm{cm}$ y $1 \Omega \times \mathrm{cm}$ en las capas sin recocido provee mayor evidencia sobre el depósito de películas delgadas mediante la técnica y parámetros mencionados, cuya estructura está conformada por una mezcla de fases entre el $\mathrm{Sn}$ policristalino y el $\mathrm{SnO}$ amorfo, donde ésta última puede producir una mayor $\rho_{e}$ en la capa, con respecto al valor típico reportado para el $\mathrm{Sn}$. Aunque sólo la fase del Sn fue detectada en la medición de los patrones XRD (ver Figura 1). De esta forma, se ha reportado que el uso de la técnica de pulverización catódica reactiva con una presión de depósito ligeramente mayor a 2 mTorr y con una $\mathrm{O}_{\mathrm{PP}}$ en el rango de $7 \%$ a $11 \%$, puede depositar películas delgadas de SnO que exhiben una estructura amorfa y también una alta resistividad (Caraveo et al., 2013).

Como se observa en la Figura 5, es evidente la reducción en la $\rho_{e}$ de las películas cuando se aplica un TA a baja temperatura, independientemente de la $\mathrm{O}_{\mathrm{PP}}$ usada durante el depósito. Este comportamiento está relacionado con la presencia de la fase del Sn policristalino y la formación del $\mathrm{SnO}$ policristalino en la estructura de las películas recocidas entre $160{ }^{\circ} \mathrm{C}$ y 200 ${ }^{\circ} \mathrm{C}$. Donde esta mezcla de fases también fue observada en los patrones XRD obtenidos y produjo cambios en las propiedades y parámetros ópticos.

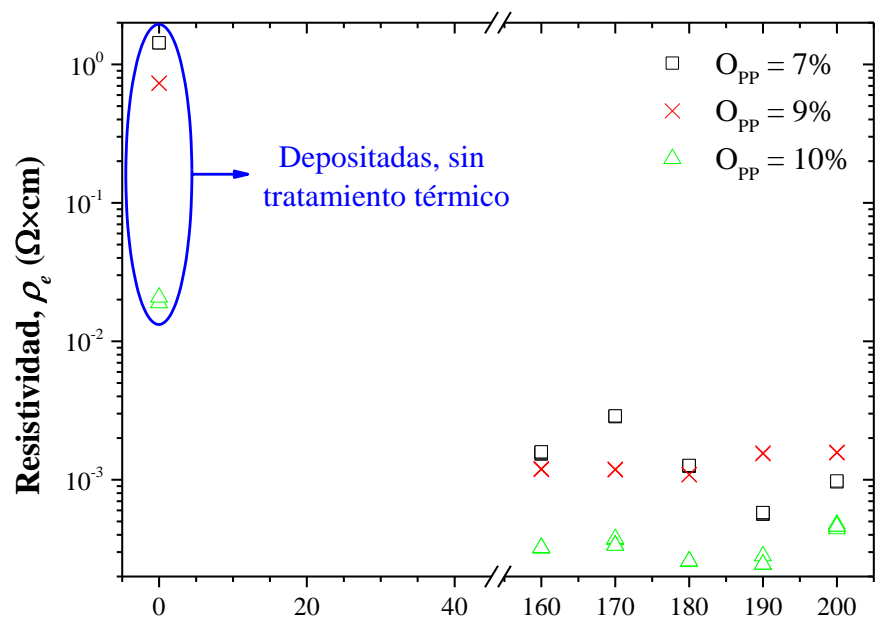

Temperatura del tratamiento $\left({ }^{\circ} \mathrm{C}\right)$

Figura 5: Dependencia de $\rho_{e}$ en las películas delgadas obtenidas con respecto a la temperatura del TA, bajo las diferentes condiciones de OPP.

Aunque no se tiene una tendencia bien definida de la variación de la $\rho_{e}$ con respecto a la temperatura del TA, se puede destacar el caso de las películas depositadas bajo una condición 
de $\mathrm{O}_{\mathrm{PP}}$ al $9 \%$ y recocidas a $190^{\circ} \mathrm{C}$ y a $200^{\circ} \mathrm{C}$, las cuales presentan una mayor $\rho_{e}$ que en los demás casos estudiados. Esto coincide con la mayor diferencia del porcentaje de $C_{i}$, donde al 9\% de $\mathrm{O}_{\mathrm{PP}}$ la diferencia entre el $C_{i}$ calculado para el plano (110) del SnO tetragonal y el $C_{i}$ determinado para el plano (200) del Sn tetragonal es del $8 \%$ y del $14 \%$ para un TA a 190 ${ }^{\circ} \mathrm{C}$ y a $200{ }^{\circ} \mathrm{C}$, respectivamente.

Se ha demostrado que es posible obtener una capa cuya estructura está compuesta por la fase del SnO puro, empleando una presión de depósito desde 1.5 mTorr hasta 2 mTorr en un rango entre $13 \% \leq \mathrm{O}_{\mathrm{PP}} \leq 15 \%$, la cual presenta propiedades de transporte tipo- $p$, pero que no provee las condiciones apropiadas para incrementar la movilidad de huecos (Caraveo et al., 2013). Sin embargo, una película policristalina de SnO que presenta un contenido de $\mathrm{Sn}$, o una mezcla de fases, ha mostrado una conductividad tipo- $p$, pero también exhibe los mayores valores de movilidad de huecos para este óxido metálico semiconductor (Caraveo et al., 2013), (Fortunato et al., 2010).

Principalmente, el origen de la conductividad tipo- $p$ del $\mathrm{SnO}$ se debe a las vacancias de estaño $\left(\mathrm{V}_{\mathrm{Sn}}\right)$, aunque también se ha sugerido que el oxígeno intersticial puede contribuir a la generación de huecos (Luo et al., 2012). De esta forma, el depósito de capas delgadas por pulverización catódica reactiva bajo condiciones enriquecidas en $\mathrm{Sn}$ puede favorecer la formación de una mayor densidad de defectos o $\mathrm{V}_{\mathrm{Sn}}$, lo cual promueve el incremento en la generación de huecos libres (Caraveo et al., 2013), (Luo et al., 2012). Sin embargo, el contenido de $\mathrm{Sn}$ debe ser controlado debido a que el exceso puede producir una mayor concentración de defectos, como estaño intersticial y/o dislocaciones, que incrementen la dispersión de los portadores y reduzcan la movilidad; o bien, una segregación del exceso de $\mathrm{Sn}$ hacia las fronteras de grano del $\mathrm{SnO}$ puede producir un comportamiento de conductividad ambipolar en el semiconductor (Li et al., 2018), (Luo et al., 2012), (Nomura et al., 2011).

\section{Conclusiones.}

Se depositaron películas delgadas con una combinación de fases de estaño metálico ( $\mathrm{Sn}$ ) tetragonal y monóxido de estaño ( $\mathrm{SnO}$ ) amorfo mediante pulverización catódica reactiva con magnetrón de DC a una presión de trabajo de 3 mTorr y una presión parcial de oxígeno en el rango entre $7 \%$ y $10 \%$. El análisis de los patrones de difracción por rayos-x (XRD), la reflectividad por rayos-x, la transmitancia óptica, la composición por espectroscopia por rayos-x de energía dispersiva y las mediciones de resistividad eléctrica proporcionan evidencia de la transición de una fase de Sn dominante a una fase de $\mathrm{SnO}$ predominante, inducida tanto por un proceso de tratamiento térmico a baja temperatura $\left(\leq 200{ }^{\circ} \mathrm{C}\right)$ como por el control de la presión parcial de oxígeno.

Aquellas películas delgadas, depositadas bajo una condición de $\mathrm{O}_{\mathrm{PP}}$ al $9 \%$ y tratadas térmicamente entre $190{ }^{\circ} \mathrm{C}$ y $200{ }^{\circ} \mathrm{C}$, presentaron una estructura con una combinación de fases entre el Sn y el $\mathrm{SnO}$, donde los picos de mayor intensidad en los patrones XRD exhiben una orientación hacia los planos (200) y (101) del Sn, y hacia el plano (110) del $\mathrm{SnO}$, que resultaron en un coeficiente relativo de textura entre el $18 \%$ y el $33 \%$ para el Sn, y entre el $37 \%$ y el $41 \%$ para el $\mathrm{SnO}$. El espesor de las capas que se obtuvieron en estas condiciones fue de $75 \pm 5 \mathrm{~nm}$, con un índice de refracción de $\sim 1.95$ y un coeficiente de extinción alrededor de -0.04 para una longitud de onda del haz incidente de $632.8 \mathrm{~nm}$, presentando una transmitancia óptica máxima del $36 \%$ dentro de la región del es- pectro UV/Vis de medición y exhibiendo un ancho de banda óptico de $\sim 2.83 \mathrm{eV}$. Adicionalmente, se determinó una densidad promedio de $6.3 \mathrm{gr} / \mathrm{cm}^{3}$ en dichas películas, una rugosidad superficial promedio de $0.75 \mathrm{~nm}$, así como un contenido de estaño de $\sim 7$ at $\%$ y de oxígeno de $\sim 13$ at $\%$, lo cual produjo una resistividad eléctrica alrededor de $1 \mathrm{~m} \Omega \times \mathrm{cm}$.

De esta forma, acorde a la evidencia presentada con la caracterización y análisis realizados sobre las películas depositadas mediante pulverización catódica reactiva con magnetrón de DC y sobre aquellas que también han sido tratadas térmicamente, además de los argumentos anteriormente expuestos, es posible determinar que las capas delgadas obtenidas al $9 \%$ de $\mathrm{O}_{\mathrm{PP}}$ y recocidas a 190 ${ }^{\circ} \mathrm{C}$ o $200{ }^{\circ} \mathrm{C}$ exhiben propiedades estructurales, ópticas, composicionales y eléctricas que podrían permitir el transporte de huecos $\mathrm{y}$, por tal motivo, posiblemente sean implementadas como capa activa tipo- $p$ en dispositivos semiconductores de película delgada con un tipo de estructura metal-dieléctrico-semiconductor.

\section{Agradecimientos}

Este trabajo fue financiado en parte por el Consejo Nacional de Ciencia y Tecnología (CONACyT) a través del Proyecto 237213 en México. Especialmente, S. I. Garduño agradece al programa del CONACyT "Cátedras CONACyT para Jóvenes Investigadores". S. Martínez agradece a CONACyT por el apoyo financiero a través de la beca 925106. Los autores agradecen el apoyo del Ing. Luis Abad por la preparación y limpieza de los sustratos utilizados. Agradecemos al M.C. Francisco J. Hernández por el depósito de las películas delgadas caracterizadas. De igual manera, un agradecimiento al M.C. Adolfo Tavira por las mediciones de XRD y XRR.

\section{Referencias}

Allen, J. P., et al., (2013). Understanding the defect chemistry of tin monoxide. Journal of Materials Chemistry C 1, 48, 8194-8208. DOI: 10.1039/C3TC31863J

Baco, S., et al., (2012). Study on Optical Properties of Tin Oxide Thin Film at Different Annealing Temperature. Journal of Science and Technology 40, $1,61-72$.

Banerjee, A. N., Chattopadhyay, K. K., (2005). Recent developments in the emerging field of crystalline p-type transparent conducting oxide thin films. Progress in Crystal Growth and Characterization of Materials 50, 13, 52-105. DOI: 10.1016/j.pcrysgrow.2005.10.001

Batzill, M., Diebold, U., (2005). The surface and materials science of tin oxide. Progress in Surface Science 79, 2-4, 47-154. DOI: 10.1016/j.progsurf.2005.09.002

Caraveo, J. A., et al., (2013). Record mobility in transparent p-type tin monoxide films and devices by phase engineering. ACS Nano 7, 6, 5160-5167. DOI: $10.1021 / \mathrm{nn} 400852 \mathrm{r}$

De Barros, A. R. X., (2014). Development of p-type oxide semiconductors based on tin oxide and its alloys: application to thin film transistors. Ph.D. dissertation, Faculdade de Ciências e Tecnologia, Universidade Nova de Lisboa, Portugal.

Fortunato, E., et al., (2010). Transparent p-type $\mathrm{SnO}_{x}$ thin film transistors produced by reactive rf magnetron sputtering followed by low temperature annealing. Applied Physic Letters 97, 5, 1-4. DOI: 10.1063/1.3469939

Fortunato, E., et al., (2012). Oxide semiconductor thin-film transistors: A review of recent advances. Advanced Materials 24, 22, 2945-2986. DOI: 10.1002/adma.201103228

Golovashkin, A. I., Motulevich, G. P., (1965). Optical properties of Tin at helium temperatures. Sovietic Physics JETP 20, 1, 46-49.

Guzman, D., et al., (2018). Fully patterned p-channel SnO TFTs using transparent $\mathrm{Al}_{2} \mathrm{O}_{3}$ gate insulator and ITO as source and drain contacts. Semiconductor Science and Technology 33, 3, 1-8. DOI: 10.1088/13616641/aaa7a6 
Guzman, D., et al., (2018). Optical properties of p-type $\mathrm{SnO}_{x}$ thin films deposited by DC reactive sputtering. Journal of Materials Science: Materials in Electronics 30, 1366-1373. DOI: 10.1007/s10854-018-0406-1

$\mathrm{Li}$, Y., et al., (2018). Ambipolar $\mathrm{SnO}_{x}$ thin-film transistor achieved at high sputtering power. Applied Physics Letters 112, 18, 1-4. DOI: $10.1063 / 1.5022875$

Liang, L. Y., et al., (2010). Phase and Optical Characterizations of Annealed SnO Thin Films and Their p-Type TFT Application service Phase and Optical Characterizations of Annealed SnO Thin Films and Their p-Type TFT Application. Journal of Electrochemical Society 157, 6, 598-603. DOI: $10.1149 / 1.3385390$

Liu, Q., et al., (2015). Tunable crystallographic grain orientation and Raman fingerprints of polycrystalline $\mathrm{SnO}$ thin films. Journal of Materials Chemistry C 3, 5, 1077-1081. DOI: 10.1039/C4TC02184C

Lorenz, M., et al., (2016). The 2016 oxide electronic materials and oxide interfaces roadmap. Jornal of Physics D: Applied Physics 49, 43, 1-53. DOI: 10.1088/0022-3727/49/43/433001

Luo, H., et al., (2012). Structural, Chemical, Optical, and Electrical Evolution of $\mathrm{SnO}_{x}$ Films Deposited by Reactive rf Magnetron Sputtering. ACS Applied Materials and Interfaces 4, 5673-5677. DOI: 10.1021/am301601s

Meyer, M., (2001). Ab initio pseudopotential calculation of the equilibrium structure of tin monoxide. Physical Review B 64, 4, 1-9. DOI: 10.1103/PhysRevB.64.045119

Nomura, K., et al., (2004). Room-temperature fabrication of transparent flexible thin-film transistors using amorphous oxide semiconductors. Nature 432, 7016, 488-492. DOI: 10.1038/nature03090

Nomura, K., et al., (2011). Ambipolar Oxide Thin-Film Transistor. Advanced Materials 23, 30, 3431-3434. DOI: 10.1002/adma.201101410

Pan, X. Q., Fu, L., (2001). Tin oxide thin films grown on the (1012) sapphire substrate. Journal of Electroceramics 7, 35-46. DOI: 10.1023/A:1012270927642

Park, J. W., et al., (2019). A Review of Low-Temperature Solution-Processed Metal Oxide Thin-Film Transistors for Flexible Electronics. Advanced Functional Materials 30, 20, 1-40. DOI: 10.1002/adfm.201904632

Shan, F., et al., (2016). High-mobility p-type NiOx thin-film transistors processed at low temperatures with $\mathrm{Al}_{2} \mathrm{O}_{3}$ high-k dielectric. Journal of Material Chemistry C 40, 9438-9444. DOI: 10.1039/C6TC02137A

Tauc, J., Menth, A., (1972). States in the Gap. Journal of Non-Cristalline Solids 8-10, 569-585. DOI: 10.1016/0022-3093(72)90194-9

Wang, Z., et al., (2016). Recent Developments in p-Type Oxide Semiconductor Materials and Devices. Advanced Materials 28, 20, 3831-3892. DOI: 10.1002/adma.201503080

Wu, M.-H., et al., (2019). Submicrometer p-Type SnO Thin-Film Transistors Fabricated by Film Profile Engineering Method. IEEE Transactions on Electron Devices 66, 4, 1766-1771. DOI: 10.1109/TED.2019.2897813

Yang, T., et al., (2015). Preparation and characterization of p-type transparent conducting $\mathrm{SnO}$ thin films. Materials Letters 139, 39-41. DOI: 10.1016/j.matlet.2014.10.040

Zhong, C.-W., et al., (2016). Impact of thermal oxygen annealing on the properties of tin oxide films and characteristics of p-type thin-film transistors. Japanese Journal of Applied Physics 55, 1-5. DOI: 10.7567/JJAP.55.016501

\section{Apéndice A.}

En la Figura A se exhiben las imágenes de la morfología superficial que fueron obtenidas mediante SEM. Únicamente se muestran aquellas que corresponden a las películas que fueron depositadas con las distintas condiciones de $\mathrm{O}_{\mathrm{PP}}$ y de las capas que también fueron tratadas térmicamente a $200{ }^{\circ} \mathrm{C}$, con el propósito de observar su superficie y analizar su composición por EDS. En la parte baja de la Figura A se muestra la escala de referencia para todas las imágenes $(7 \mu \mathrm{m})$, las cuales fueron adquiridas con un voltaje de aceleración de 10 kV y una magnificación de 5000x.

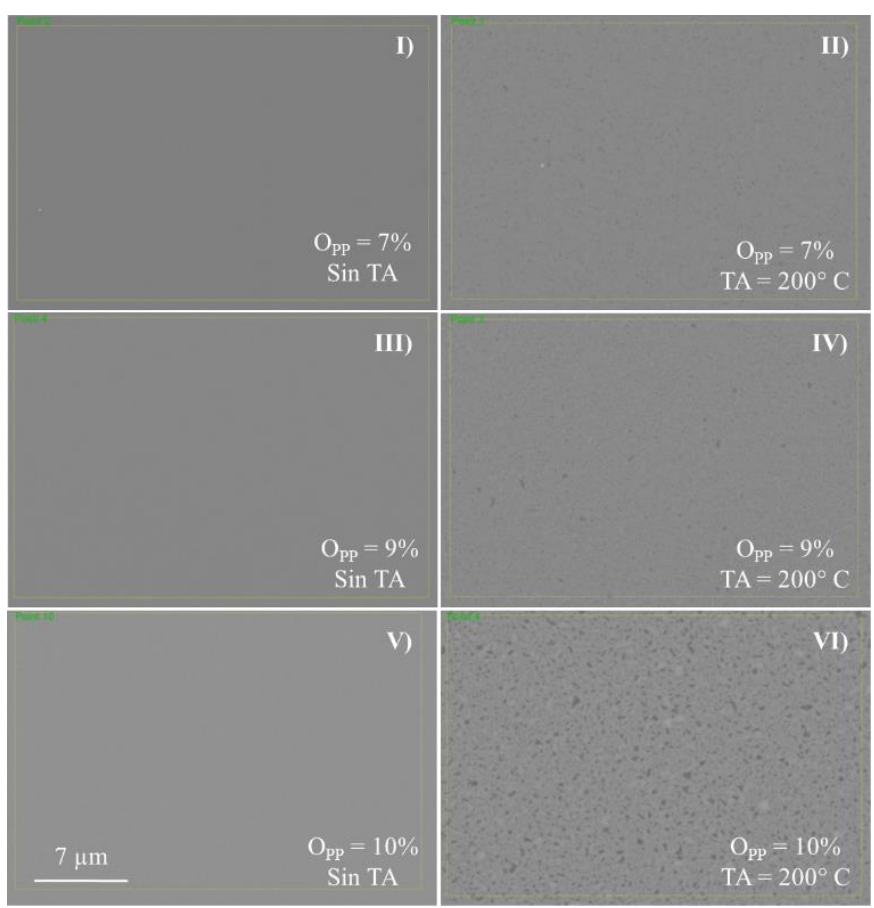

Figura A: Imágenes SEM mostrando la morfología superficial de las películas delgadas depositadas a una OPP del: I-II) $7 \%$, III-IV) $9 \%$ y V-VI) $10 \%$; y aquellas tratadas térmicamente a $200{ }^{\circ} \mathrm{C}$ (II, IV y VI). 